\title{
Bidding with Securities: Comment
}

\author{
YeOn-Koo Che ${ }^{1} \quad$ Jinwoo $\mathrm{KIM}^{2}$
}

${ }^{1}$ Columbia University, Economics Department; E-mail: yeonkooche@gmail.com

${ }^{2}$ Yonsei University, School of Economics; E-mail: jikim@yonsei.ac.kr. 


\begin{abstract}
Peter DeMarzo, Ilan Kremer and Andrzej Skrzypacz (2005, henceforth DKS) analyzed auctions in which bidders compete in securities. They show that a steeper security leads to a higher expected revenue for the seller, and also use this to establish the revenue ranking between standard auctions. In this comment, we obtain the opposite results to DKS's by assuming that a higher return requires a higher investment cost. Given this latter assumption, steeper securities are more vulnerable to adverse selection, and may thus yield lower expected revenue, than flatter ones.
\end{abstract}


Peter DeMarzo, Ilan Kremer and Andrzej Skrzypacz (2005, henceforth DKS) analyzed auctions in which bidders compete in securities, i.e., the winning bidder's payment includes a share of cash flow or (ex-post) value generated from the auctioned object. With attention restricted to "feasible" securities, ${ }^{1}$ their main finding concerns the role of the "steepness" of securities in determining the seller's revenue. They show that the steeper the payment to the seller as a function of the realized value, the higher is the seller's expected revenue. A shift from a security, say a debt, to a steeper one, say equity or call option, "flattens" the surplus accruing to a bidder as a function of his future realized value, and this levels the competitive gaps between bidders. Hence, the competition becomes intensified. ${ }^{2}$

In this comment, we wish to add to DKS' analysis a caveat - that the adverse selection problem should be an important part of security and auction design consideration. Unlike cash bids, security bids are difficult to evaluate when the seller does not know the buyer's exact type. For instance, a $40 \%$ share of an asset managed by a buyer may be less valuable than a $30 \%$ share of the same asset managed by a different buyer, if the latter is much more competent. Lacking such information, a seller may fall victim to adverse selection, choosing a wrong bidder. This problem has been noted by several authors such as William Samuelson (1987), Simon Board (2007) and Charles Zheng (2001) in the context of a fixed security design. Our concern here is its relevance for security design: We show that the adverse selection problem may lead to the rankings of alternative security designs and auction formats that are quite different from, and in fact opposite to, those found in DKS. In particular, under a reasonable circumstance, a steeper security is more vulnerable to adverse selection, and could result in a poorer revenue performance, than a flatter security.

To illustrate, suppose there are two buyers, 1 and 2. Buyer $i=1,2$ has a project which requires initial investment of $x_{i}$ and generates a (gross) return of $x_{i}+v_{i}$. Suppose $v_{1}>v_{2} \geq 0$ and $x_{1}>x_{2}$. That is, buyer 1's project generates a higher return (so is efficient to select) but requires a higher investment than buyer 2's project. This assumption is reasonable. A financially distressed or bankrupt firm will more likely turn profitable again under the management willing to infuse more cash, for instance. Or a firm that can generate a greater value from a under-performing target is more likely to have a higher opportunity cost (e.g., of pursuing other target) or stand-alone values. The assumption is equally compelling in other contexts, such as the government sale of oil leases.

\footnotetext{
${ }^{1} \mathrm{~A}$ security is feasible if the shares accruing to the payee and payer are both nondecreasing in the realized gross return. As DKS observe, all standard financial claims satisfy this monotonicity condition.

${ }^{2}$ This insight originates from Robert Hansen (1985) and is further developed by Matthew Rhodes-Kropf and S. Viswanathan (2000).
} 
Assume a second-price auction is used. Suppose first the buyers bid in standard debt. Then, buyer $i$ is willing to bid up to $v_{i}$. Hence, buyer 1 will win and must pay $v_{2}$. Deterministic return means that the revenue for the seller is $v_{2}$, the same as that under the cash auction.

Suppose next the buyers bid in the standard equity. Buyer $i$ will then bid a share $s_{i}$ that will leave him with zero profit: $\left(1-s_{i}\right)\left(v_{i}+x_{i}\right)-x_{i}=0$, or

$$
s_{i}=\frac{v_{i}}{x_{i}+v_{i}} .
$$

The efficient buyer is no longer assured of winning. Buyer 1 wins if $\frac{v_{1}}{x_{1}}>\frac{v_{2}}{x_{2}}$, but he loses if $\frac{v_{1}}{x_{1}}<\frac{v_{2}}{x_{2}}$. In the former case, the seller's revenue is $\frac{x_{1}+v_{1}}{x_{2}+v_{2}} v_{2}>v_{2}$, so it is higher than that under the cash or debt auction. But in the latter case, the seller receives

$$
\frac{x_{2}+v_{2}}{x_{1}+v_{1}} v_{1}<v_{2},
$$

which is less than what either cash or debt auction would bring.

Consider finally the steepest design, a call option. This is equivalent to the buyers competing for a debt issued by the seller. Again the logic of the second-price auction implies that buyer $i$ will ask for repayment rate of $x_{i}$, which will just pay for his investment cost. Since the low bid wins in this case, bidder 2 wins always! As a result, the seller receives

$$
\max \left\{v_{2}+x_{2}-x_{1}, 0\right\}
$$

which is the least among all revenues from all other security auctions, regardless of who wins in the equity auction. ${ }^{3}$

In what follows, we generalize this observation, establishing first the sense in which a steeper security is more vulnerable to adverse selection than a flatter security and second how the adverse selection problem affects the seller's revenue.

\section{Model}

Each bidder's type is represented by his project, indexed by the net expected return, $v \in[\underline{v}, \bar{v}]$. The investment project with return $v$ requires initial investment $x(v)$, where $x(\underline{v}) \geq 0$ and

\footnotetext{
${ }^{3}$ When $\frac{v_{1}}{x_{1}}<\frac{v_{2}}{x_{2}}$, the seller's revenue from equity auction is$$
\frac{x_{2}+v_{2}}{x_{1}+v_{1}} v_{1}>\max \left\{v_{2}+x_{2}-x_{1}, 0\right\}
$$

since $\frac{v_{1}}{x_{1}+v_{1}}$ is increasing in $v_{1}$ and since the LHS equals the middle term when $v_{1}$ falls to $v_{2}+x_{2}-x_{1}$.
} 
$x^{\prime}(\cdot) \geq 0$. The project $v$ generates gross return $Z$, which is distributed over $[\underline{z}, \bar{z}]$, where $\underline{z} \leq x(\underline{v})$. Without loss, we can redefine $v=\mathbb{E}[Z \mid v]-x(v)$ to be the net expected return. Clearly, it is efficient for the buyer with the highest project return to be selected. Adverse selection arises when the seller fails to select the buyer efficiently.

The ensuing model is precisely the same as DKS, except that we allow the investment $\operatorname{cost} x(v)$ to rise with the project type $v$. This feature is quite plausible, as motivated in the introduction, and also serves to identify the different extents to which alternative security designs and auction formats are vulnerable to adverse selection.

As with DKS, we consider an ordered set of feasible securities $\mathcal{S}=\{S(s, Z): s \in[0,1]\}$ : For all $v, \frac{\partial \mathbb{E}[S(s, Z) \mid v]}{\partial s}>0$. For simplicity, we write $\mathbb{E}[S(s, Z) \mid v]$ as $E S(s, v)$ and its derivatives as $E S_{v}(s, v)$ and $E S_{s}(s, v)$. Also, as in DKS, we compare two ordered sets of securities, $\mathcal{S}^{1}$ and $\mathcal{S}^{2}$, in terms of steepness: $\mathcal{S}^{1}$ is steeper than $\mathcal{S}^{2}$ if for all $S^{1} \in \mathcal{S}^{1}$ and $S^{2} \in \mathcal{S}^{2}$, we have $E S_{v}^{1}\left(s^{1}, v\right)>E S_{v}^{2}\left(s^{2}, v\right)$ whenever $E S^{1}\left(s^{1}, v\right)=E S^{2}\left(s^{2}, v\right)$. Note that $\mathcal{S}^{1}$ being steeper than $\mathcal{S}^{2}$ implies that for all $S^{1} \in \mathcal{S}^{1}$ and $S^{2} \in \mathcal{S}^{2}, E S^{1}(s, v)$ and $E S^{2}(s, v)$ are single-crossing: Whenever $E S^{1}(s, v)=E S^{2}(s, v)$ for some $v$, we have $E S^{1}\left(s, v^{\prime}\right)>(<) E S^{2}\left(s, v^{\prime}\right)$ if $v^{\prime}>(<) v$.

\section{Ranking Security Designs}

\subsection{Second-Price Auctions}

We first consider a second-price auction in which each bidder submits a security from an ordered set $\mathcal{S}$ and the seller selects a bidder with highest security (highest $s$ ), who then pays the second highest security. In a weakly dominant equilibrium, a type $v$ submits a bid $s(v) \in[0,1]$ such that

$$
E S(s(v), v)-v=0
$$

Adverse selection manifests itself as the failure of monotonic equilibrium strategies. In particular, we focus on the possibility of extreme adverse selection where bidders employ decreasing bidding strategies. To this end, differentiate both sides of (1) with $v$ to obtain

$$
E S_{s}(s, v) s^{\prime}(v)+E S_{v}(s, v)-1=0
$$

or

$$
E S_{s}(s, v) s^{\prime}(v)=1-E S_{v}(s, v) .
$$


Since $E S_{s}(s, v)>0, s(\cdot)$ will be decreasing if and only if

$$
E S_{v}(s, v)>1 \text { whenever } E S(s, v)=v .
$$

It follows from this that a steeper security is more vulnerable to adverse selection than a flatter design:

Proposition 1. Let $s^{1}(\cdot)$ and $s^{2}(\cdot)$ denote the equilibrium strategies under securities $\mathcal{S}^{1}$ and $\mathcal{S}^{2}$, respectively. Suppose that $\mathcal{S}^{1}$ is steeper than $\mathcal{S}^{2}$. Then, if $s^{2}(\cdot)$ is decreasing, $s^{1}(\cdot)$ is decreasing also. Also, if $s^{1}(\cdot)$ is increasing, $s^{2}(\cdot)$ too is increasing.

Proof. Since $s^{1}(\cdot)$ and $s^{2}(\cdot)$ constitute the equilibrium under $\mathcal{S}^{1}$ and $\mathcal{S}^{2}$, respectively, we have $E S^{1}\left(s^{1}(v), v\right)=v=E S^{2}\left(s^{2}(v), v\right)$ for all $v$. Then,

$$
E S_{v}^{1}\left(s^{1}(v), v\right)>E S_{v}^{2}\left(s^{2}(v), v\right)>1
$$

where the first inequality follows from $\mathcal{S}^{1}$ being steeper than $\mathcal{S}^{2}$ and the second from $s^{2}(\cdot)$ being decreasing. Thus, $s^{1}(\cdot)$ is decreasing. A similar argument can be used to show that if $s_{1}(\cdot)$ is increasing, $s_{2}(\cdot)$ is also increasing.

The next proposition establishes a convenient sufficient condition for adverse selection to arise for some security design. It states that any security design as steep as, or steeper than, standard equity will induce a decreasing equilibrium, if the investment cost $x(v)$ increases at a rate faster than the net return $v$.

Proposition 2. A second-price equity auction induces a decreasing (resp. increasing) equilibrium bidding strategy if $x(v) / v$ is increasing (resp. decreasing) in $v$.

Proof. Letting $s$ denote the equity share, (1) becomes

$$
E S(s(v), v)=s(v) \mathbb{E}[Z \mid v]=s(v)(v+x(v))=v
$$

or

$$
s(v)=\frac{v}{v+x(v)}=\frac{1}{1+x(v) / v},
$$

which is increasing (resp. decreasing) if $x(v) / v$ is decreasing (resp. increasing).

We now explore the revenue consequence of adverse selection.

Proposition 3. Suppose that $\mathcal{S}^{1}$ is steeper than $\mathcal{S}^{2}$. Letting $s^{1}(\cdot)$ denote the equilibrium bidding strategy under $\mathcal{S}^{1}$, if $s^{1}(\cdot)$ is decreasing, then the seller's revenue is lower with $\mathcal{S}^{2}$ than with $\mathcal{S}^{1}$. 
Proof. We show that the desired revenue ranking holds in the ex-post sense. Fix a value profile at $v_{1}, \cdots, v_{n}$ and let $v_{(r)}$ denote the $r$-th highest value. Since $s^{1}(\cdot)$ is decreasing, a winner under $\mathcal{S}^{1}$ pays $E S^{1}\left(s^{1}\left(v_{(n-1)}\right), v_{(n)}\right)<E S^{1}\left(s^{1}\left(v_{(n)}\right), v_{(n)}\right)=v_{(n)}$. First, we consider the case in which under $\mathcal{S}^{2}$, a winner's value is $v>v_{(n)}$ and the second highest bidder's value is $\tilde{v}$. Then, the winner pays $E S^{2}\left(s^{2}(\tilde{v}), v\right)$, which is higher than what is paid by the winner under $\mathcal{S}^{1}$ since

$$
E S^{2}\left(s^{2}(\tilde{v}), v\right)>E S^{2}\left(s^{2}(\tilde{v}), v_{(n)}\right) \geq E S^{2}\left(s^{2}\left(v_{(n)}\right), v_{(n)}\right)=v_{(n)}>E S^{1}\left(s^{1}\left(v_{(n-1)}\right), v_{(n)}\right) .
$$

Second, consider the case in which, under $\mathcal{S}^{2}$, a winner's value is $v_{(n)}$ and the second highest bidder's value is $\tilde{v}$. In this case, the winner under $\mathcal{S}^{2}$ pays $E S^{2}\left(s^{2}(\tilde{v}), v_{(n)}\right) \geq E S^{2}\left(s^{2}\left(v_{(n-1)}\right), v_{(n)}\right)>$ $E S^{1}\left(s^{1}\left(v_{(n-1)}\right), v_{(n)}\right)$, where the second inequality follows from the facts that $\mathcal{S}^{1}$ is steeper than $\mathcal{S}^{2}, \operatorname{ES}^{1}\left(s^{1}\left(v_{(n-1)}\right), v_{(n-1)}\right)=E S^{2}\left(s^{2}\left(v_{(n-1)}\right), v_{(n-1)}\right)=v_{(n-1)}$, and that $v_{(n)}<v_{(n-1)}$.

Not only is a steeper security design more vulnerable to adverse selection than a flatter one, but the former entails lower expected revenue than the latter, even when the latter too suffers adverse selection. The reason is that the steeper design magnifies the competitive differences of the bidders than a flatter design when decreasing equilibrium strategies are employed under both designs.

As noted by DKS, a call option is the steepest, and standard debt is the flattest, among all feasible securities. Further, a cash payment is even flatter than a standard debt. Combining Propositions 2 and 3, we then arrive at rather surprising implications much in contrast with DKS:

Corollary 1. Suppose $x(v) / v$ is increasing in $v$. Then, for a second-price auction,

(i) cash or debt yields higher expected revenue than equity or any securities steeper than standard equity.

(ii) a call option yields the lowest expected revenue among all feasible securities.

While the condition involves a restriction, part (ii) may likely hold under a weaker condition. For instance, the result will hold whenever a call option auction induces a decreasing equilibrium.

\subsection{First-Price Auctions}

We now establish analogous results about security designs under a first-price auction. To this end, we assume that $v$ is symmetrically distributed across bidders, following a distribution 
$F:[\underline{v}, \bar{v}] \rightarrow[0,1]$ with a density $f(v)>0$ for all $v \in(\underline{v}, \bar{v})$. We focus on a symmetric equilibrium bidding strategy $s_{F}(\cdot)$ that is differentiable.

As before, we first establish the sense in which a steeper security design is more vulnerable than a flatter one to adverse selection. All proofs not provided here appear in Appendix.

Proposition 4. Suppose that $\mathcal{S}^{1}$ is steeper than $\mathcal{S}^{2}$. Suppose also that the first-price auction with $\mathcal{S}^{2}$ induces a decreasing equilibrium bidding strategy. Then, the equilibrium of the firstprice auction with $\mathcal{S}^{1}$, if exists, must also be decreasing.

As before, adverse selection affects revenue more adversely for a steeper security design than a flatter one, which stands in contrast with the finding of DKS.

Proposition 5. Suppose that $\mathcal{S}^{1}$ is steeper than $\mathcal{S}^{2}$ and the first-price auction with $\mathcal{S}^{2}$ induces a decreasing equilibrium strategy. Then, the seller revenue is lower with $\mathcal{S}^{2}$ than with $\mathcal{S}^{1}$ whenever the latter admits an equilibrium.

\section{Ranking Auction Formats}

In this section, we study how adverse selection can affect the ranking of standard auction formats. We first show that there is a sense in which the first-price auction is more prone to adverse selection than a second-price auction:

Proposition 6. Suppose the equilibrium bidding strategy of the second-price auction is decreasing. Then, any equilibrium bidding strategy of the first-price auction, if exists, must also be decreasing.

This difference in the two standard auction formats has consequences for the seller's expected revenue. We now show that, much in contrast to DKS's finding, whenever the adverse selection plagues both formats under a given feasible security, a second-price auction yields a higher expected revenue than a first-price auction.

To this end, following DKS, we call an ordered set, $\mathcal{S}$, of securities super-convex if every $S \in$ $\mathcal{S}$ is steeper than any security obtained from a (nontrivial) convex combination of securities in $\mathcal{S}$, and they are convex if it is equal to its convex hull.

Proposition 7. Suppose that $\mathcal{S}$ is super-convex (resp. convex) and the second-price auction induces a decreasing equilibrium bidding strategy. Then, the first-price auction generates a lower (resp. the same) expected revenue than the second-price auction whenever the former admits an equilibrium. 
As noted in DKS, call options are super-convex, and equity is convex. Combining the results established thus far yields the following implications.

Corollary 2. Suppose $x(v) / v$ is increasing in $v$.

(i) A standard (i.e., first-or second-price) cash or debt auction yields higher expected revenue than does any standard auction with equity or any securities steeper than equity.

(ii) A first-price auction with call options yields lower expected revenue than does any standard auction with any securities.

Proof. Given the condition, a second-price equity auction induces a decreasing equilibrium (Proposition 2). Since equity is convex, by Proposition 7, first- and second-price auctions are revenue equivalent under equity. Part (i) then follows from Propositions 3 and 7.

We next prove part (ii). Note first that Propositions 1 and 2 and imply that a second-price auction in call options induces a decreasing equilibrium. Then, by Proposition 6, a first-price auction with call options induces a decreasing equilibrium. It then follows from Proposition 5 that a first-price auction with call options yields lower revenue than does a first-price auction with any regular securities. Next, since call options are super-convex (as noted by DKS), Proposition 7 implies that a first-price auction with call options yields lower revenue than a second-price auction with call options, which, by Corollary 1, in turn yields lower revenue than a second-price auction with any securities.

\section{Concluding Remarks}

We conclude our comment with two remarks. First, we have focused on the extreme form of adverse selection in which the bidders adopt decreasing bidding strategies. This implicitly assumes that the seller commits to a given auction rule. If she makes no such commitment but rather selects the winner ex post optimally based on her inference from equilibrium strategies, then a separating equilibrium will unravel, so a decreasing equilibrium will not arise. While it is difficult to describe the resulting equilibrium precisely, our analysis seems relevant even in this latter case, for a couple of reasons. First, whenever the equilibrium involves a decreasing strategy in our model, the worst type can profitably mimic the equilibrium strategy of any type. This means that the bidder will not be selected efficiently, so adverse selection is unavoidable, in equilibrium. In this sense, focusing on the circumstance entailing a decreasing equilibrium (under our exogenous rule) serves as a useful proxy for the severity of adverse 
selection even in this case. Second, adverse selection appears to have a serious revenue consequence for the seller even when she behaves ex post optimally. No matter how the seller chooses the winner, given adverse selection (under our exogenous rule), the seller's revenue must be low enough to leave the worst type with positive rents. ${ }^{4}$ For this reason, adverse selection should be taken seriously in auction/security design.

Second, the precise implications of adverse selection for security and auction design depend on the underlying model. Nevertheless, the particular model we have considered seems plausible. Further, its main feature - that a higher return corresponds to a higher investment cost - lends itself to a standard moral hazard interpretation. That is, an insight similar to those developed here will apply if the winning bidder's return depends on his costly effort. A steep security design could suppress the incentive for the efforts and thus could reduce the surplus accruing to the seller.

\section{Appendix}

Proof of Proposition 4. Let $s_{F}^{i}(\cdot)$ denote the equilibrium strategy of the first-price auction with $\mathcal{S}^{i}$ and $s^{i}(\cdot)$ that of the second-price auction so that $s^{i}(\cdot)$ is a solution of (1) with $\mathcal{S}^{i}$. As assumed, $s_{F}^{2}(\cdot)$ is decreasing. The standard argument can be used to show that there is no atom in the support of $s_{F}^{i}(\cdot), i=1,2$.

SteP 1. $\bar{v}$ is the unique minimizer of $s_{F}^{1}(\cdot)$.

Proof. Suppose for a contradiction that $s_{F}^{1}(\cdot)$ is minimized at some $v^{\prime}<\bar{v}$ so that $v^{\prime}$ has to obtain zero payoff at equilibrium under $\mathcal{S}^{1}$ since there is no atom. Since $s_{F}^{2}(\cdot)$ is decreasing and thus $\bar{v}$ obtains zero equilibrium payoff with $\mathcal{S}^{2}$, it must be that $E S^{2}\left(s_{F}^{2}(\bar{v}), \bar{v}\right)=\bar{v}=$ $E S^{1}\left(s^{1}(\bar{v}), \bar{v}\right)$. (Recall $s^{i}(\cdot)$ denotes the equilibrium strategy of the second-price auction with security $\mathcal{S}^{i}$, satisfying (1).) Given this, $\mathcal{S}^{1}$ being steeper than $\mathcal{S}^{2}$ implies that

$$
E S^{2}\left(s_{F}^{2}(\bar{v}), v\right)>E S^{1}\left(s^{1}(\bar{v}), v\right) \text { for all } v<\bar{v}
$$

\footnotetext{
${ }^{4}$ This again follows from the observation that if our exogenous auction rule induces a decreasing equilibrium, the worst type can profitably mimic the equilibrium strategy of any type under the endogenous selection rule.
} 
So, we have

$$
\begin{aligned}
v^{\prime}-E S^{1}\left(s_{F}^{1}(\bar{v}), v^{\prime}\right) & \geq v^{\prime}-E S^{1}\left(s^{1}(\bar{v}), v^{\prime}\right) \\
& >v^{\prime}-E S^{2}\left(s_{F}^{2}(\bar{v}), v^{\prime}\right) \\
& >v^{\prime}-E S^{2}\left(s_{F}^{2}\left(v^{\prime}\right), v^{\prime}\right) \\
& \geq 0=v^{\prime}-E S^{1}\left(s_{F}^{1}\left(v^{\prime}\right), v^{\prime}\right),
\end{aligned}
$$

where the first inequality follows from $s_{F}^{1}(\bar{v}) \leq s^{1}(\bar{v})$, the second from $(3)$, the third from $s_{F}^{2}(\cdot)$ decreasing, the fourth from $s_{F}^{2}\left(v^{\prime}\right)$ being the equilibrium bid for $v^{\prime}$ with $\mathcal{S}^{2}$, and the last equality from $v^{\prime}$ earning zero equilibrium payoff with $\mathcal{S}^{1}$. The above inequality results in $E S^{1}\left(s_{F}^{1}(\bar{v}), v^{\prime}\right)<E S^{1}\left(s_{F}^{1}\left(v^{\prime}\right), v^{\prime}\right)$ or $s_{F}^{1}(\bar{v})<s_{F}^{1}\left(v^{\prime}\right)$, contradicting that $s_{F}^{1}(\cdot)$ is minimized at $v^{\prime}$.

To simplify notation, $s_{F}^{1}(\cdot)$ is denoted as $s_{F}(\cdot)$ from now on. Then, Step 1 implies $s_{F}(\bar{v})<$ $s_{F}(\underline{v})$, so the proof will be complete if it can be shown that $s_{F}^{\prime}(v) \neq 0$ for all $v \in(\underline{v}, \bar{v})$, which is established in the next two steps.

STEP 2. Let $V_{0}=\left\{v \in(\underline{v}, \bar{v}): s_{F}^{\prime}(v)=0\right.$ and $v$ is a local minimum $\}$. Then, $V_{0}=\emptyset$.

Proof. Suppose that $V_{0}$ is not empty. One can then find $v_{0} \in V_{0}$ such that $s_{F}\left(v_{0}\right) \leq s_{F}\left(v^{\prime}\right)$ for all $v^{\prime} \in V_{0}$. One can also find some $v_{1} \in\left(v_{0}, \bar{v}\right)$ such that $s_{F}\left(v_{1}\right)=s_{F}\left(v_{0}\right)$ and $s_{F}^{\prime}\left(v_{1}\right)<0 .{ }^{5}$ Consider a downward deviation by $v_{1}$ to slightly lower $s$ and mimic $v_{1}+\epsilon$ with small $\epsilon>0$. It is straightforward that as $\epsilon \rightarrow 0$, the marginal cost from decrease in the winning probability is

$$
d_{-}:=(n-1) f\left(v_{1}\right) F^{n-2}\left(v_{1}\right)\left[v_{1}-E\left(s_{F}\left(v_{1}\right), v_{1}\right)\right]
$$

while the marginal benefit from decrease in the expected payment is

$$
d_{+}:=-\left(1-F\left(v_{1}\right)\right)^{n-1} s_{F}^{\prime}\left(v_{1}\right) E S_{s}\left(s_{F}\left(v_{1}\right), v_{1}\right) .
$$

For this deviation to be unprofitable, we must have $d_{-} \geq d_{+}$. Consider now an upward deviation by $v_{1}$ to slightly raise $s$ and mimic $v_{1}-\epsilon$ for small $\epsilon>0$. We will then be able to find some $\epsilon_{1}(\epsilon), \epsilon_{2}(\epsilon)>0$ such that $s_{F}\left(v_{0}-\epsilon_{1}(\epsilon)\right)=s_{F}\left(v_{0}+\epsilon_{2}(\epsilon)\right)=s_{F}\left(v_{1}-\epsilon\right)$. With this deviation, the winning probability is equal to

$$
w(\epsilon):=\sum_{k=0}^{n-1}\left(\begin{array}{c}
n-1 \\
k
\end{array}\right)\left(F\left(v_{0}+\epsilon_{2}(\epsilon)\right)-F\left(v_{0}-\epsilon_{1}(\epsilon)\right)\right)^{k}\left(1-F\left(v_{1}-\epsilon\right)\right)^{n-1-k} .
$$

\footnotetext{
${ }^{5}$ This is possible since $\bar{v}$ is a unique minimizer of $s_{F}(\cdot)$
} 
Thus,

$$
\begin{aligned}
\lim _{\epsilon \rightarrow 0} \frac{w(\epsilon)-w(0)}{\epsilon} \geq & \lim _{\epsilon \rightarrow 0} \frac{\left(1-F\left(v_{1}-\epsilon\right)\right)^{n-1}-\left(1-F\left(v_{1}\right)\right)^{n-1}}{\epsilon} \\
& +\lim _{\epsilon \rightarrow 0} \frac{(n-1)\left(F\left(v_{0}+\epsilon_{2}(\epsilon)\right)-F\left(v_{0}-\epsilon_{1}(\epsilon)\right)\right)\left(1-F\left(v_{1}-\epsilon\right)\right)^{n-2}}{\epsilon} \\
> & (n-1) f\left(v_{1}\right) F^{n-2}\left(v_{1}\right) .
\end{aligned}
$$

Here, the strict inequality holds true since

$$
\begin{aligned}
& \lim _{\epsilon \rightarrow 0} \frac{(n-1)\left(F\left(v_{0}+\epsilon_{2}(\epsilon)\right)-F\left(v_{0}-\epsilon_{1}(\epsilon)\right)\right)\left(1-F\left(v_{1}-\epsilon\right)\right)^{n-2}}{\epsilon} \\
= & (n-1) f\left(v_{0}\right)\left(\epsilon_{1}^{\prime}(0)+\epsilon_{2}^{\prime}(0)\right)\left(1-F\left(v_{1}\right)\right)^{n-2}>0,
\end{aligned}
$$

which is in turn due to the fact that $\epsilon_{1}^{\prime}(0)$ and $\epsilon_{2}^{\prime}(0)$ are positive since $v_{0}$ is a local minimizer of $s_{F}(\cdot){ }^{6}$ By (4), the marginal increase in the winning probability from the upward deviation is greater than $(n-1) f\left(v_{1}\right) F^{n-2}\left(v_{1}\right)$, which means that the associated marginal benefit is greater than $d_{-}$. Clearly, the marginal cost from increase in the expected payment is equal to $d_{+}$. Considering $d_{-} \geq d_{+}$, however, this implies that the upward deviation is profitable. $\|$

Given Step 2, that $s_{F}^{\prime}(v) \neq 0$ for all $v \in(\underline{v}, \bar{v})$ can be established if we rule out the case in which $s_{F}(\cdot)$ is hump-shaped, which leads to Step 3.

STEP 3. There is no $v \in(\underline{v}, \bar{v})$ at which $s_{F}(\cdot)$ achieves a (global) maximum.

Proof. Suppose to the contrary that there is a global maximizer $v_{m} \in(\underline{v}, \bar{v})$. One must be then able to find some $v_{1} \in\left(v_{m}, \bar{v}\right)$ such that $s_{F}\left(v_{1}\right)=s_{F}(\underline{v})$ and $s_{F}^{\prime}\left(v_{1}\right)<0$. Considering a downward deviation by $v_{1}$, one can obtain the expressions for associated marginal benefit and cost that are the same as $d_{+}$and $d_{-}$defined in Step 2. Also, it must be that $d_{-} \geq d_{+}$. Then, a similar argument to the one that resulted in (4) above, can be used to show that an upward deviation will lead to the marginal increase in winning probability, which is greater than $(n-1) f\left(v_{1}\right) F^{n-2}\left(v_{1}\right)$. So, the marginal benefit from the upward deviation is greater than $d_{-}$while the marginal cost is equal to $d_{+}$, which implies that the upward deviation is profitable. ||

Proof of Proposition 5. According to Proposition 4, given the condition, the equilibrium bidding strategy under $\mathcal{S}^{1}$, whenever it exists, must be decreasing. We then mimic the proof of Proposition 1 in DKS. To that end, let $s_{F}^{i}(v)$ and $U^{i}(v)$ denote the type $v$ 's equilibrium bid and equilibrium payoff, respectively, in the first-price auction with $\mathcal{S}^{i}$.

\footnotetext{
${ }^{6}$ More precisely, $\epsilon_{1}(\epsilon)=v_{0}-s_{F}^{-1}\left(s_{F}\left(v_{1}-\epsilon\right)\right)$ and thus $\epsilon_{1}^{\prime}(0)=\frac{-s_{F}^{\prime}\left(v_{1}\right)}{s_{F}^{\prime}\left(v_{0}\right)}=\infty$, and similarly for $\epsilon_{2}^{\prime}(0)$.
} 
Claim 1. If $U^{2}(v)<U^{1}(v)$ for some $v<\bar{v}$, then $U^{2}\left(v^{\prime}\right)<U^{1}\left(v^{\prime}\right)$ for all $v^{\prime}<v$.

Proof. Suppose not. Then, there must be some $\hat{v}<\bar{v}$ such that $U^{1}(\hat{v})=U^{2}(\hat{v})$ and $\frac{d U^{1}(\hat{v})}{d v} \geq \frac{d U^{2}(\hat{v})}{d v}$. This implies that $E S^{1}\left(s_{F}^{1}(\hat{v}), \hat{v}\right)=E S^{2}\left(s_{F}^{2}(\hat{v}), \hat{v}\right)$ and, by the envelope theorem,

$$
\frac{d U^{1}(\hat{v})}{d v}=(1-F(\hat{v}))^{n}\left[1-\frac{\partial E S^{1}\left(s_{F}^{1}(\hat{v}), \hat{v}\right)}{\partial v}\right] \geq(1-F(\hat{v}))^{n}\left[1-\frac{\partial E S^{2}\left(s_{F}^{2}(\hat{v}), \hat{v}\right)}{\partial v}\right]=\frac{d U^{2}(\hat{v})}{d v}
$$

or $\frac{\partial E S^{1}\left(s_{F}^{1}(\hat{v}), \hat{v}\right)}{\partial v} \leq \frac{\partial E S^{2}\left(s_{F}^{2}(\hat{v}), \hat{v}\right)}{\partial v}$, which is a contradiction since $\mathcal{S}^{1}$ is steeper than $\mathcal{S}^{2} . \|$

Now consider the equilibrium of the first-price auction under $\mathcal{S}^{2}$ with a modified distribution which is the same as $F(\cdot)$ except that the support is truncated at $\bar{v}-\epsilon$ for small $\epsilon$ and there is a mass equal to $1-F(\bar{v}-\epsilon)$ at $\bar{v}-\epsilon$. Letting $U_{\epsilon}^{2}(\cdot)$ denote the payoff for this equilibrium, we have $U_{\epsilon}^{2}(\bar{v}-\epsilon)=0<U^{1}(\bar{v}-\epsilon)$. Note that the above claim still holds between $U_{\epsilon}^{2}(\cdot)$ and $U^{1}(\cdot)$ with $\bar{v}$ being replaced by $\bar{v}-\epsilon$. Thus, we have $U_{\epsilon}^{2}(v)<U^{1}(v)$ for all $v \leq \bar{v}-\epsilon$. By making $\epsilon$ converge to zero, we conclude that the buyers' payoffs are higher with $\mathcal{S}^{1}$, which implies that the seller's revenue is higher with $\mathcal{S}^{2}$, as desired.

Proof of Proposition 6. Recall $s(\cdot)$ denote the solution of $(1)$. Clearly, $s_{F}(v) \leq s(v)$ for all $v$.

As in Step 1 of Proposition 4, we first prove that the highest type $\bar{v}$ must be the unique minimizer of $s_{F}(\cdot)$. Suppose to the contrary that there is some $v^{\prime}<\bar{v}$ at which $s_{F}(\cdot)$ is minimized and that the interim equilibrium payoff is zero or $v^{\prime}=E S\left(s_{F}\left(v^{\prime}\right), v^{\prime}\right)$ so $s_{F}\left(v^{\prime}\right)=$ $s\left(v^{\prime}\right)$. However, the fact that $s(\cdot)$ is decreasing and $s_{F}\left(v^{\prime}\right)=s\left(v^{\prime}\right)$ implies by (2) that $v-$ $E S\left(s_{F}\left(v^{\prime}\right), v\right)<0$ for some $v$ in the neighborhood of $v^{\prime}$, which leads to a contradiction that

$$
v-E S(s(v), v) \leq v-E S\left(s_{F}(v), v\right) \leq v-E S\left(s_{F}\left(v^{\prime}\right), v\right)<0,
$$

since $s(v) \geq s_{F}(v) \geq s_{F}\left(v^{\prime}\right)$. Thus, we conclude that $\bar{v}$ is the unique minimizer of $s_{F}(\cdot)$. The rest of proof then follows the same line of argument as that in Step 2 and Step 3 of the proof of Proposition 4 and is thus omitted. I

Proof of Proposition 7. Note that according to Proposition 6, any equilibrium bidding strategy of the first-price auction must be decreasing. Then, the proof follows the same line of argument as the proof of Proposition 5, for the super-convexity of $\mathcal{S}$ implies that

$$
\frac{\partial E S\left(s_{F}(v), v\right)}{\partial v}>\frac{\partial \mathbb{E}\left[E S\left(s\left(v^{\prime}\right), v\right) \mid v^{\prime}>v\right]}{\partial v}
$$

if $E S\left(s_{F}(v), v\right)=\mathbb{E}\left[E S\left(s\left(v^{\prime}\right), v\right) \mid v^{\prime}>v\right]$. 
The proof of revenue equivalence between the first- and second-price auctions with convex securities follows from the observation that if $\mathcal{S}$ is convex, then the inequality in (5) becomes an equality, which makes all the inequalities in the proof of Proposition 5 into equalities. I

\section{References}

[1] Board, Simon. "Bidding into the Red: A Model of Post-Auction Bankruptcy," Journal of Finance, 2007 LXII, 2695-2723.

[2] DeMarzo, Peter, Kremer, Ilan and Skrzypacz, Andrzej. "Bidding with SecuritiesAuctions and Security Design," American Economic Review, 2005, 95, 936-959.

[3] Hansen, Robert G. "Auctions with Contingent Payments, American Economic Review, 1985, 75, 862-65.

[4] Rhodes-Kropf, Matthew, and Viswanathan S. "Financing Auction Bids," Journal of Finance, 2000, 55, 1807-49.

[5] Samuelson, William. "Auctions with Contingent Payments: Comment," American Economic Review, 1987, 77, 740-45.

[6] Zheng, Charles Z. "High Bids and Broke Winners," Journal of Economic Theory, 2001, 100, 129-171 